\title{
HANDLING PATTERN OF PESTICIDES IN VEGETABLES: A CASE STUDY OF BHAKTAPUR
}

\author{
Naba Raj Budhathoki ${ }^{1}$, Pushpa Raj Acharya ${ }^{2}$ and Dhruva Karki ${ }^{3}$ \\ ${ }^{1}$ Department Of Science and Humanities, Khwopa Enginering College, Purbanchal University, Bhaktapur, Nepal \\ ${ }^{2}$ Nepal Academy of Science and Technology, Lalitpur, Nepal \\ ${ }^{3}$ Cental Department of English, Tribhuvan University, Kathmandu, Nepal
}

\begin{abstract}
This research work explores handling practices of pesticides used in vegetables on the part of farmers in Bhaktapur. Specifically, it attempts to investigate the contribution of pesticides applicators to overall mishandling of pesticides. Data for this work were collected in 2018 from four locations of Bhaktapur. Primary data were collected from 123 vegetable farmers and direct observation of 42 vegetable farms based in the selected zones using questionnaire. Discussions with experts and pilot study with farmers were conducted to improve the questionnaire and provide factual data. Frequency distributions, Means, Chi-square tests were utilised for data analysis. The results of the study show that vegetable farmers were applying pesticides, without caring much about the others, in vegetable production. They frequently depended upon local sellers and neighbours to choose the best pesticide to apply. On average, pesticide handling practices at any point seemed inappropriate. Factors that influence farmers' handling practices include education, training and practical consciousness. The study indicated that vegetable farm women have higher level of exposure of pesticide risking a long term health impacts. The results of the study underscore the need of educational interventions for promoting safety during each step of pesticide handling and the responsibility of the farmers towards the society.
\end{abstract}

Keywords: Vegetable growers, Application of pesticides, Pesticides handling, Health risks

\section{Introduction}

\subsection{History of Pesticides in Nepal}

The term "pesticide" recapitulates a broad range of chemical compounds, including insecticides, fungicides, herbicides, rodenticides, molluscicides, nematicides and plant growth regulators. Dichlorodiphenyl trichloroethane (DDT) is the first ever pesticide applied in Nepal in 1952. The introduction of other synthetic insecticides organophosphate (OP) insecticides in the 1960s, carbamates in the 1970s and pyrethroids in the1980s--, and the introduction of herbicides and

\footnotetext{
*Corresponding author: Naba Raj Budhathoki

Department of Science and Humanities, Khwopa

Engineering College, Libali-8 Bhaktapur

Email: nrbudhathoki@yahoo.com,

(Received: $8^{\text {th }}$ Dec 2018 Accepted: $17^{\text {th }}$ April 2019
}

fungicides during the 1970s and the 1980s contributed greatly to pest control and agricultural output (Manandhar, 2006).

Nowadays, the vegetable farmers apply pesticides to destroy organisms, including insects, rodents and fungi, to protect the vegetable production and preserve the produced materials.

Since the 1960s, Nepal's government has given major emphasis on import and supply of chemical pesticides to increase agricultural production, and as a result pesticides started to be used indiscriminately and broadly throughout the country. According to WHO, $20 \%$ of the pesticides produced in the world is being used in developing countries, including Nepal. Nepal's use of pesticides (which is $142 \mathrm{~g} / \mathrm{ha}$ (ADB, cited in Sharma et al., 2012) is still very low in comparison to that of India (500 gm/ha), Japan 
(12 kg/ha), or Korea (6.6 kg/ha) (Gupta et al., 2011). This low average use could be attributed to an uneven distribution of pesticide applications in Nepal. Pesticides application is heavily concentrated in the cultivation of vegetables, mustard and cotton, and more intensive in the Terai region, Kathmandu valley and its surrounding areas where agriculture is being increasingly commercialized.

Usage of pesticides in Nepalese agriculture is regulated by Act and Law; however, law enforcement is almost absent in major vegetable growing. Nepal government has passed Plant Protection Act 1972; Plant Protection Rules 1975; Pesticide Act in 1991; Pesticide Rules 1993; Environmental Protection Act 1996 and Environmental Protection Rules in order to manage the discriminate use of pesticides (Palikhe, 2002).

Pesticide use is not static due to many factors, such as, availability of alternatives, market prices, effectiveness and pesticide availability in markets. Earlier studies have not explored sufficiently the recent use pattern of pesticides and its market system in totality. Available information does not provide information about the real status of pesticide application in Nepal. Nepal Government has started to monitor vegetable products by setting up in several locations, including Kalimati, a machine for rapid assay just separating the vegetables into three categories with three indicators --green, yellow and red. The green color means the vegetables are consumable. The yellow means they should be put in certain waiting period before consumption. Those vegetables which test red must be thrown. This is of course a positive step towards lessening the overuse of pesticides, but more is required to monitor the application of pesticides. Besides, Nepal is a member of World Trade Organization that also requires authentic data of pesticide use for the export of agricultural products.

\subsection{Pesticides in Bhaktapur}

Most of the vegetable farm lands based in Bhaktapur, which are fairly near from the main bazaar of the valley, are used for the cultivation of vegetables, like cauliflower, tomatoes, cabbage and other sagpat (green leaves). Insecticides, along with fertilizers, are heavily used in these areas. The rainy and dry seasons obviously affect vegetable production in these places. Furthermore, there is variation in temperature over the year in the study area. One of the major cities in the Kathmandu Valley, Bhaktapur, was once self-reliant in agriculture. However, at present its vegetable production has been much commercialized, and it is feeding vegetables to its inhabitants and supplying vegetables to Kalimiti Bazaar, along with several small markets in other locations of the valley to remarkable extents. Even though the application of pesticides has contributed to an increasing productivity in vegetables in Bhaktapur, the successes have unfortunately often been accompanied with problem of mishandling of pesticides (Pimentel et al., 1993).

Vegetables are important sources of micronutrients and provide farmers with higher income per hectare than cereal and root (AVRDC, 2006). Vegetable production is a year-round activity in Bhaktapur. The vegetable crops' production allows reaping several harvests within the same year, and a great combination of them, or maintaining the same crop all year long, due to their short cycle ( 1 to 5 months). Some regions of the district have specialized in the production of certain vegetable crops, such as green leaves of spinach and pumpkin in Sagbari of Thimi Municipality. Likewise, farmers in different parts of the Valley produce vegetables, considering soil, water and temperatures throughout the year. The farmers' handling of pesticides in vegetable farming of Bhaktapur needs an in-depth study for its significant roles in providing vegetables across Kathmandu Valley.

\subsection{Issues of Pesticides Application in Vegetables}

Although there are some disagreements on the degree of risks owing to pesticides, it appears that people are increasingly being concerned that improper pesticide use can cause hazardous impacts on human health and environmental quality. It has come to limelight from the WHO Report, millions of farm workers are estimated to suffer from pesticide poisoning every year. At least, 20,000 farm workers die from the exposure each year, and most of them in the developing countries, like Nepal. The rampant use of these chemicals, 
believing in the adage, "if little is good, a lot more will be better" has rendered havoc in humans and other life forms.

Vegetables receive chemical pesticides fairly at a greater rate than that of other crops. In the meantime, vegetables form part of a healthy diet because they provide vitamins and minerals, too. Biotic and abiotic factors are among the major constraints of vegetable production. Both of the quality and quantity of vegetables are highly affected by the presence of pests in them. Therefore, pesticides use is one of many tools in practice for effective protection of vegetables along with other crops from weeds, insects and diseases, and agricultural pesticide use is rapidly increasing each year in many of developing countries, including Nepal and India.

This study focuses on vegetables because the pesticide use is particularly high in these crops, which are susceptible to a large number of arthropod pests and diseases while consumers prefer unblemished pest free vegetables. The production and productivity of vegetables have been increasingly significant in recent years primarily because of excessive availability of pesticides (Shrestha et al., 2010). Without pesticides, farmers would have lost their plants, fruits and vegetables round the year. Moreover, they would not have saved seeds for producing plants in their farms.

As there is no much study published in such a scale to find the relation between many factors in handling the pesticides from the beginning to the very end, this study is worthy. The findings of the study could provide a background for the government and the concerned bodies while planning for educational programs and trainings for the sake of vegetable farmers and eventually agricultural farmers to protect them from the occupational hazards due to the use of pesticides.

Although accepting pesticides as 'dangerous things,' farmers in Nepal, stuck on an agrochemical treadmill, are unwilling or unable to give them up. Pesticides could be a problem in both the cases-whether they are misused or over used. The worse problem may arise due to invisibility of long term effects to the non-target species, environment and finally to human beings at large. There have been few studies in the study area to estimate such effects from the concerned bodies. There is a considerable gap of data related with health effects, environmental contamination and specific incidence of pesticide poisoning, which have marred the monitoring activity.

It is very interesting to note that various existing data show the pesticide use seems to be low in developed countries, but pesticide poisoning seems to be high as compared to the amount of pesticide used. This kind of difference signifies that something is wrong in the handling of pesticides them on the part of several stakeholders, including the applicators. They are the ones who remain closer with the chemical pesticides for a long time from the time of buying through applying to the time of consuming them.

Researchers have concluded that farm workers in developing countries will continue to use pesticides in increasing quantities because of the absence of alternatives to pesticides, unawareness of the sustainability of pesticide use, and the lack of strong enforcement of regulations and laws on pesticide application (Wilson and Tisdell, 2001). In the same lines, vegetable farmers are suspected to cause harms on themselves as applicators due to negligence and more importantly on others, near or far, due to the lack of practical consciousness. Since the farmers' handling of pesticides in vegetable farming of Bhaktapur needs an in-depth study for its significant roles in providing vegetables across Kathmandu Valley, this study tries to explore the ways vegetable growers handle pesticides right from the time of purchasing to the time of disposal of containers

\section{Materials and Methods}

The present research work could be seen basically as three tasks: Desk Study, Field Study and Data Analysis. During the period of desk study, various literatures, including books and papers were reviewed. From them, updated information on the handling of pesticides was garnered. Field study was carried out in the selected sites to collect the information about the handling of pesticides and safety. 


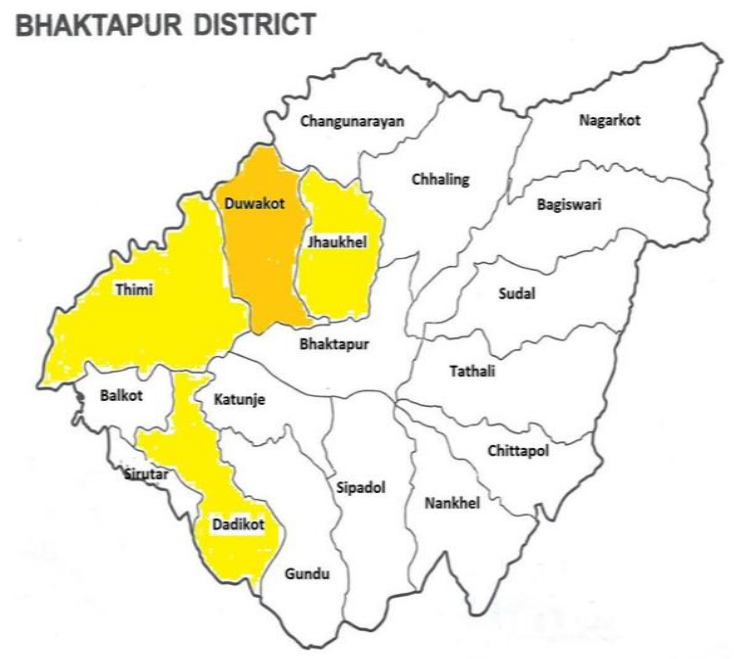

Fig. 1. Selected zones for study

This study was carried out to assess the existing knowledge of vegetable growers, as far as handling of the pesticides is concerned, from the very beginning, i.e., from the point of deciding to buy a particular pesticide till the end, i.e., to the point of disposing the container through questionnaire interview with 123 vegetable growers identified and direct observation of 42 vegetable farms based in the selected zones. The vegetable growers using pesticides for agricultural enhancement were only chosen. Finally, the vegetable farmers' self-reported pesticide compliance behaviors were analyzed. Regarding the methodology, this study takes an interdisciplinary approach--combining law, sociology, psychology and public administration scholarships.

Four well known zones for vegetables--Jhaukhel, Duwakot, Dadhikot, Thimi-- in Bhaktapur (Fig 1) were selected for the study. It is the smallest district of the country by area, covering area of 138.46 square kilometer. This district lies $27^{0} 36^{\prime}$ to $27^{\circ} 44^{\prime}$ northern latitude and $85^{\circ} 32^{\prime}$ to $85^{\circ} 32^{\prime}$ eastern longitude and bordering with Kavrepalanchok, Kathmandu, and Lalitpur districts. With its geophysical areas of hills and plains, Bhaktapur is considered a suitable area for vegetable farming. Moreover, Thimi, Jhaukhel, Duwakot and Dadhikot are considered major areas for the production of commercial vegetables. The selection of these places was based on high concentration of vegetable farms. Although constant encroachment of the fertile land has altered the vegetation and greatly depleted the fertility of the soil, these four are the existing major vegetable producing areas, comparatively.

Sampling sites were in vegetable production regions of the selected zones and determined by use of multi-location random sampling. The sample size for each region was determined based on its share of vegetable production. The regions surveyed and sample sizes include 21 farmers in Thimi, 49 in Duwakot, 34 in Dadhikot, and 38 in Jhaukhel. The study was carried out from January to April 2018. For the questionnaire, proposed questions were initially developed in English language and sent once to various pesticides' specialists for their critique and suggested additions. After responses were received from the specialists, their suggestions and comments were utilized in developing the final semi-structured (also considered mixed questionnaire) questionnaire consisting of both closed and open-ended questions. The questionnaire contained 52 main questions. Ten farmers participated in the pre-study (pilot study) to insure farmer comprehension of typical questions and the ability of respondents to administer it. Their responses were used to improve the final copy. The survey questionnaire instrument was developed to document the current indiscriminate use and improper application of pesticides by vegetable farmers in Bhaktapur. While executing the questionnaire during each interview, the questions were either explained in Nepali or Nepal Bhasa when required in the majority of the cases as most of the respondents were illiterate or just literate.

All the data were collected from face to face interviews after the informed consent. Although sampling was random, an effort was made to ensure that the selected farmers represented different locations, age groups, farm sizes and literacy levels. The target of the study was explained to these respondents through the personal communication. This was done in order to ensure their cooperation, which was very important for the study. In addition, follow-up phone calls and visits, when necessary, to the respondents were made to extract any information needed even after the real interview. Their farms ranged from small (half ropani) and family operated and without utilizing advanced farming technology to the big and employees operated ones (20 ropani) with the utilization of 
limited modern technology.

All data from standardized questionnaire were coded, entered, and then analyzed using the Statistical Package for Social Sciences (SPSS). In this study, we assessed the farmers' knowledge and its association with practice of pesticide use.

\section{Results}

\subsection{Demographics and Socioeconomic Status of Participants}

Survey responses were obtained from 123 respondents representing four major vegetable producing zones in Bhaktapur. The highest percentage of responses was obtained from Duwakot (39\%), meanwhile the lowest responses' percentage was recorded from Jhaukhel with only $15 \%$. While the data revealed a satisfactory crosssection through the district, the overall average farmers' age was estimated at 43 , in which the youngest farmer was 24 years old and the eldest was 68 years old. Splitting the farmers' age into groups showed that the majority of the farmers were in their forties, while the minority of the farmers were in their sixties. On an average, survey respondents were middle-aged.

Vegetable growers were discovered to be either Hindu (83\%) or Buddhist (7\%). Eighty one percent of the farmers in the study were Newars while the Chhetri were $12 \%$, and Brahmins were $7 \%$. Others were not noted in the survey sites. Most of the people involved in vegetables liked to put themselves in working class of social strata. Likewise, high percentage of vegetable growers used Newari (78\%) with the family at home while the rest used Nepali. Among the respondents, $80 \%$ consumed tobacco in one form or the other.

General Information of Participants:

The categories of the vegetable farmers varied between age, sex, family status, and level of education. Eighty percent of vegetable farmers were men, and $20 \%$ were women. Most of them (77\%) were household heads which lead the decision of using pesticides. The increasing involvement of young and educated vegetable growers seems to be promising for the safe use of pesticides. However, the percentage of elderly population (aged 60 and above) was the least, i.e., $10.78 \%$ only.

Table 1: General information about participants:

\begin{tabular}{lc}
\hline Level of Education & Number (\%) \\
\hline Illiterate & 39 \\
Literate & 39 \\
SLC & 30 \\
Bachelor's level & 6 \\
Master's level & 9 \\
\hline
\end{tabular}

Similarly, maximum percentage of the people were illiterate i.e., $32 \%$ of the total respondents, and $32 \%$ of them were under SLC, $25 \%$ finished their +2 level education, whereas very few in the range of (510) $\%$ were found to have had university level education (Table 1). More importantly, almost none was found to have field specific formal education or field specific training of remarkable level although majority of the farmers had the vegetable farming as major source of income. And educational level of schooling, the most dominant among the farmers with $78 \%$ was either illiterate or just literate, i.e. under Grade 8.

Most of the farmers in the study area were running vegetable farms as the only occupation for maintaining daily expenses although some are doing it as subsidiary one. Out of the total number of vegetable farmers interviewed, $96 \%$ produced vegetables for market and $4 \%$ produced for both the market and home consumption.

\subsection{Pesticide Application Practices}

A very high percentage $(90 \%)$ of the farmers purchased pesticides from retailers available in local markets, and believed that they would still apply pesticides in the coming years. Farmers who received advice from shopkeepers tended to use more pesticides in amount. More than half of the farmers $(61 \%)$ applied pesticides when necessary, while $30 \%$ applied pesticides all year.

For pesticide application, the hand compression (usually 9-litre capacity) and the knapsack sprayer (16-litre capacity) were very commonly used. In the study, $86 \%$ of the respondents used knapsack sprayer (Fig 2). In the absence of a sprayer, locally made brooms and jhari were also used. Similarly, in the absence of a duster, pesticide dust was spread 
over plants and soil surface by hand.

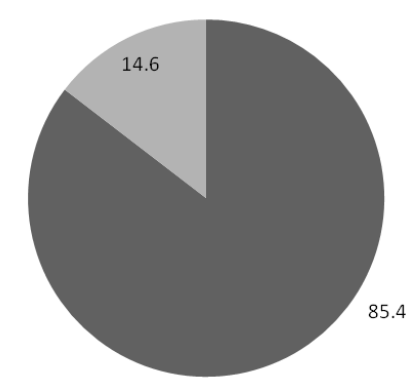

Fig. 2. Pie chart of equipment used for application of pesticides

There was no facility of water to wash after work for more than $45 \%$ of the respondents. Majority of them (93\%) used the soap and water for hand washing and any spillage. Almost 56\% of the farmers applied pesticides without caring wind direction, which could minimize inhalation of pesticides and skin contact. Whoever considered the wind direction while spraying did not postpone the spraying even when it was windy. The present study showed that almost $60 \%$ of total interviewed farmers used some of the safety measures, like using the mouth covers such as masks or cloths on mouth, the gloves, etc (Fig 3). Almost none used all set of Pesticides Protection Equipment (PPE), including coverall, in combination.

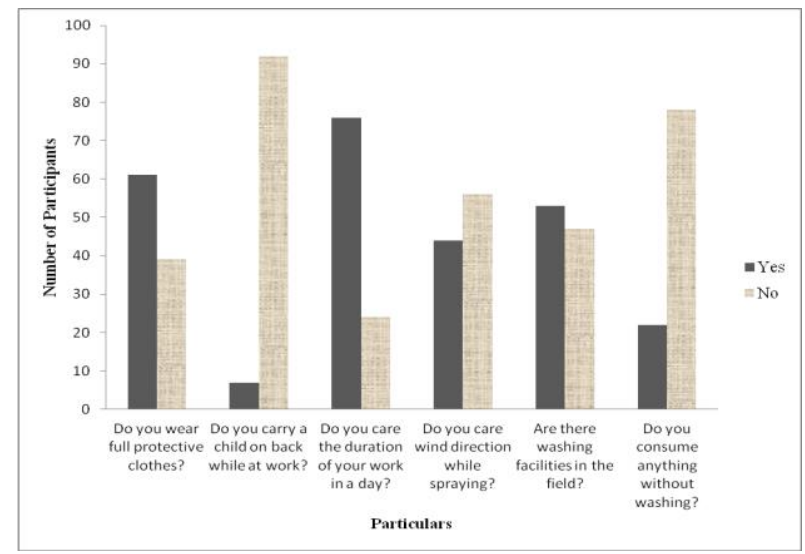

Fig. 3. Bar diagram of major activities during the application of pesticides

It is notable that $7 \%$ of the people carried a child on back while applying pesticides in the field causing gross exposure to the innocent child while $20 \%$ were female participants. In the observation male applicator, as usual, had female companion, usually his wife. More than $24 \%$ of the respondents did not even care duration of work in a day. Worse is that $20 \%$ did not mind eating things without proper washing of hands or other body parts. Nor did any keep a diary to record required information and field experiences about the application of pesticides

\subsection{Vegetable Growers' Knowledge of Pesticide Application}

Respondents reported that they were very interested in both quality and quantity of the vegetables they grow. In addition, more than $90 \%$ of farmers said they had concern about human health. However, almost $73 \%$ of the respondents do not read the pesticide label and did not much care about the pesticides' level of toxicity before using it.

One-third of the workers read the label on the pesticide packet either themselves or seeking help. But only less than 3\% followed the instructions given to the specific pesticide. The workers often related the toxicity of pesticides to the odour of the chemical and more pungent ones are considered as most toxic. The scientific categorization based on colour code is rarely understood. Sixty-three per cent of the farm workers knew that pesticides with different levels of toxicity are available in the market, starting from relatively safe ones to highly toxic. But $99.5 \%$ of them could not understand the toxicity level reading the colour code on the bottle. However, almost all of the applicators did not take food or smoke while spraying the pesticides.

Some components of protective covering of body parts was adopted by 71 per cent of the respondents while spraying. In many cases, it was mainly the full- sleeved wind-cheater. But it was noted during the field observation that some rolled up their sleeves while doing spraying/mixing. Majority of respondents wore mask or tied a piece of cloth around the nose. A mere 1 per cent used some form of eye protection (e.g. ordinary spectacles, which were actually there even otherwise), though most of them reported eye irritation after spraying. . Furthermore, $78 \%$ of them haven't got access to services for consultaion. In case of small scale farmers doing home garden, they don't personally feel like not being that serious on any matters regarding the application of pesticides. 


\subsection{Concern about the Environment and Human Health}

Negligible number of respondents stored pesticides in special store, while 56\% stored them in locked area and 39\% in open area randomly. In addition, Forty percent of the participants stored the pesticides in open area, and $25 \%$ even kept the pesticides in other containers rather than the original container.

Their sanitation activities were noted poor; they even reported not maintaining bath one time in one week. They usually washed hands and legs as usual with soap. Likewise, remarkable number of the participants did not change clothes completely. Even pesticide containers were found to be used for secondary purposes after general washing by $43 \%$ of the vegetable farmers of the study area. About $42 \%$ of the respondents washed the bottle/ sprayer in the nearby water bodies.

Table 2: Mode of disposing empty container

\begin{tabular}{lll}
\hline \multicolumn{1}{c}{ Activity } & Frequency & Percent \\
\hline Bury or Burn & 6 & 4.9 \\
Burn & 33 & 26.8 \\
Bury & 12 & 9.8 \\
Return & 3 & 2.4 \\
Thrown & 69 & 56.1 \\
\hline Total & 123 & 100.0 \\
\hline
\end{tabular}

The majority of the farmers (69\%) threw empty containers in open spaces; $33 \%$ burn, and $12 \%$ bury them (Table 2). Farmers appear quite confused about the disposal and they were doing this seeing another farmer do the same in the neighbourhood.

The majority of vegetable farmers who indicated noncompliance usually harvested vegetables within two or three days after pesticide spraying. In the study, a little less than that (43\%) did not follow the rule of waiting period especially for those vegetables which they don't consume but sell to others. Instead, small-scale farmers who have modest needs for pesticides on their small size farms will purchase small amounts decanted into secondary containers including soft drink bottles, which are particularly hazardous. Thirty percent of the surveyed people grew the vegetables different ways for selling and for using in family. In the interview some people also reported they don't care about the waiting period although they know the waiting period roughly for a particular pesticide

\section{Discussion}

The study explores on perceptions and pesticides use practices of vegetable farmers by portraying the situation of pesticide use of vegetable farmers based in Bhaktapur. The vegetable growing activities are led by males in the survey zones. Similarly, in Brazil overall farming was dominated by males as reported in a study (Recena et al., 2006). The data revealed a satisfactory cross-section throughout Bhaktapur district. Eighty percent of men seemed to be involved in the vegetable farming. At that point, the heads of family were found to be male, who decided everything about pesticide application. The male domination in the vegetable farming can be associated with cultural factors of the patriarchal Nepali society. They took the role of head and did major works, like spraying the pesticides. However, women are involved in the field itself longer hours, spending whole days, getting exposed to chemicals constantly.

Women have a particular susceptibility to pesticides due to their physiological characteristics, lifestyle, and behavior, too (Berkowitz et al., 2004). Women in the family are at a greater risk of accumulated exposure because of long working hours from an early age and multiple exposures, both at work and domestic settings, with potential contact to pesticides through working conditions, eating contaminated plants and products, washing of contaminated clothing, drinking contaminated water, and intense use of multitudes of pesticides in agriculture (Tingle et al., 2000). In an analysis of cancer among 146,000 California Hispanic women farmers, compared with the general Hispanic population, these women were more likely to develop certain types of leukemia by $59 \%$, cervical cancer by $63 \%$, uterine cancer by $68 \%$, and stomach cancer by $70 \%$ (Mills and Kwong, 2001). Studies have also documented increased incidence of miscarriages, still births, and delayed pregnancy among women farm workers and wives of men employed in pesticide mixing and spraying in agrofood industries.. The transfer of farm chemicals in 
breast milk is also a concern - it is estimated that in Delhi, India the average infant receives 12 times the acceptable level of DDT (Ransom, 2002). Clearly, the intensive use of pesticides poses great health risks for farm women and, through them their families. Their role in pesticide-related activities multiplies when vegetables are involved.

Increasing participation of young generation in vegetable farming has a positive connotation as it is expected there will be more educated people in agriculture. It will, as seen in the survey, help lessen the pesticide exposure.

Knowledge, attitudes and application patterns of the pesticides vegetable farmers in Bhaktapur were surveyed. Education matters to the knowledge of pesticides. This was evident from this study as well as in a study at Palestine (Al Saed et al., 2011). where significant association was reported good level of knowledge about pesticide and education (Table 3 ). In the study majority of the farmers were aware that pesticides would affect human health and the body through the nose. Information from training conducted by the government agencies reflects in the level of good knowledge among farmers. This finding was consistent with the present study. The educational background of the farm workers in the study area was too low. Similar results were also reported in other developing countries. Farm workers with little formal education might be at a higher risk while using pesticides, possibly due to difficulties in understanding the application instructions and safety procedures included on the product label. A high level of knowledge was recorded among the participants who had a higher education level. Vegetable farm workers with good pesticide knowledge were not as inclined as expected to use pesticides according to the recommended guidelines for protective measures, a finding that was consistent with other studies. Training has not been effective regarding the pesticide handling and growing vegetables. The data of the government sector show that huge amount of money has been spent in the training of local people in the modern agriculture and pest handling. Majority of them wanted to have field based training that should increase the production and should be practicable.
Table 3: Association between the level of education and reading label on the container or seeking help for that purpose:

\begin{tabular}{|l|c|c|c|}
\hline \multirow{2}{*}{ Education level } & \multicolumn{2}{|c|}{$\begin{array}{l}\text { Reading pesticide label } \\
\text { Or Seeking help }\end{array}$} & \multirow{2}{*}{ Total } \\
\cline { 2 - 3 } & No & Yes & \\
\hline Illiterate & 39 & 0 & 39 \\
\hline Literate & 36 & 3 & 39 \\
\hline SLC & 15 & 15 & 30 \\
\hline Bachelor & 0 & 6 & 6 \\
\hline Master & 0 & 9 & 9 \\
\hline Total & 90 & 33 & 123 \\
\hline
\end{tabular}

Most of the vegetable growers apply preventative sprays and that may be due to the lack of proper knowledge and information about pests and pesticides. Applications of pesticides beforehand in the field cause extra expenses and overuse of pesticides.

Though there is a licensing system in this sector, the sale was discovered often unregulated in the observation. The pesticides could be brought over the counter without any prescription or scientific supervision. Studies reveal that a large majority of farmers are consulting the traders for choosing the chemical and the dose, against specific symptoms. But these traders often have little technical expertise or the training support to offer scientific technical advice.

Whoever considered the wind direction while spraying did not postpone the spraying even when it was windy. This resulted in higher chances of drift, affecting the non-target population. The adoption of safety measures during and after pesticide application is very important factor for preventing against harmful impacts of pesticide. The various safety options could be use of gloves, masks, long sleeved cloth, glass, long boots etc. The present study showed that $61 \%$ of total interviewed farmers used some of the safety measures like using the mouth covers such as masks and cloths on mouth, the gloves. Almost none used all set of PPE, including coverall, in combination. This may put them in the risk of pesticides, and the health of these people is not secure of the disease caused by the inhalation, ingestion and absorption via skin. This 
may lead to occupational health hazards. Similarly, A study in the community of Culturama in Brazil shows that A great majority $(>90 \%)$ considered pesticides to be harmful to human health, but less than $20 \%$ used masks, impermeable clothes, or gloves during pesticide application ( Recena et al., 2006). Most often farmers had little knowledge of pest management as well as the consequences of pesticide use (Mahantesh and Singh, 2009), and even if they are aware of the negative health effects of pesticides, many still do not use personal protective devices (PPDs). Chi- square test of independence between wearing protective clothes and formal education indicated with $\mathrm{p}$ - value 0.005 that there exists positive association between the education and following safety measures during the spray (Fig 4).



Fig. 4. Bar diagram of wearing protective clothes as against education

Nepalese farmers were much found to use very crude method in the past while spraying chemical pesticides. They even used broom, which is actually used to clean the house and its surroundings, to spray them. However, few were found to do such practices in the study area. However, even a single example is not ignorable owing to its serious repercussions. In-depth studies indicate that farmers' high doses of pesticides cause acute effects, whereas low level of exposure for a long time lead to chronic effects (Alavanja et al., 2004).

Soap and water are used for washing hands in every case after the application of pesticides. The people are conscious about the hand washing, but the procedure of the hand washing is not satisfactory. Majority of them (93\%) used the soap and water for hand washing and any spillage both. This may clean up the pesticide traces in the hands but cannot clean up fully.

The use of containers for refilling pesticides is another potentially unsafe approach for disposal, due to the fact that it can encourage product adulteration and movement of products with misleading instructions or with no instructions at all leading to poor handling and application. This study found that few farmers disposed of their empty pesticide containers through returning them to manufacturers. Returning of empty containers to manufacturers could be a useful method for safe and economic disposal, but, in Nepal, there is no direct link between a farmer and the manufacturer. Fear is that some retailers may misuse the empty containers for decanting or repacking of adulterated products instead of returning them to manufacturers.

Minority of them kept the pesticide containers along with food items at home and did not use the empty containers for storage of food items. The findings of the study indicate that when it comes to social behavior, the care is comparatively less. About 42 per cent of the respondents washed the bottle/ sprayer in the nearby water bodies. They are poisoning the aquatic animals (Weston et al., 2004). 
Furthermore, they grow the vegetables different ways for selling and for using in family. They don't care about the waiting period although they know the waiting period roughly for a particular pesticide. For earning the money they said they should sell the vegetables before passing waiting period. If they wait they lag behind the competitor in the market and even the vegetable look wilt and consumers don't like to buy such vegetables no matter what.

\section{Conclusion}

The responses to the key factors on scientific use of pesticides have reflected that the awareness regarding the handling practices are considerably low. Vegetable growers handle toxic chemicals thinking as if they are safe enough 'Aushadhi'. The results presented here display insufficient awareness of vegetable farmers of the district towards pesticide use and a lack of appropriate knowledge on the safety measures while handling pesticides and more importantly remarkable negligence while handling pesticides for the sake of themselves and others.

Despite high literacy level among some participants, most of them do not trouble reading the instructions on the packets and follow them. Nevertheless, the results of this study supported to certain extent that the hypothesis that farm workers with good knowledge of pesticides show good practice in pesticide use. Therefore, it is strongly recommended to intensify special educational programs, legislation promoting the use of safer pesticides, and the implementation of personal protective measures; such approaches are necessary to decrease the pesticide exposure of farm workers and others alike.

More importantly informed farmers failed to think about others, it can be represented through carelessness in waiting period and throwing left over and containers haphazardly. Also, vegetable production is especially handled by the high illiteracy level farmers and the aging farming population. Moreover, low knowledge of proper use of pesticides was associated with more use of pesticide. This is reflected in overuse, misuse and abuse of chemical pesticides through the improper estimation of the timing and frequency of application, not reading labels on pesticide containers, not wearing protective equipment fully, storage of pesticide containers in home and disposal of empty pesticide containers unscientifically. Knowledge garnered from this work will support to ameliorate existing situations of pesticide application regulations.

A limitation of the present study was the inability to delve into the details of the health effects of pesticides on the applicators. More in-depth studies on the health impacts of pesticide exposure on the applicators and other stakeholders are recommended.

\section{References}

[1] Alavanja M. C., Hopkin J. A. \& Kamel F. (2004). Health Effects of Chronic Pesticide Exposure: Cancer And Neurotoxicity, Ann. Rev. Pub. Health 25: 155197.

[2] Al-Saed R., Ramlawi A. \& Salah A. (2011). A survey on utilization of agricultural pesticides in selected Palestinian districts. International J. Environ. Stud., 68: 519-529.

[3] AVRDC- Asian Vegetable Research and Development Center (2006). Vegetables matter. (http:// www.avrdc.org).

[4] Berkowitz, G. S., Wetmur, J. G., Birman-Deych, E., Obel, J., Lapinski, R. H., Godbold, J. H., \& Wolff, M. S. (2004). In utero pesticide exposure, maternal paraoxonase activity, and head circumference. Environmental Health Perspectives, 112(3), 388 -399

[5] Gupta, V. K., Gupta, B., Rastogi, A., Agarwal, S., \& Nayak, A. (2011). Pesticides removal from waste water by activated carbon prepared from waste rubber tire. Water Research, 45(13), 4047-4055.

[6] Mahantesh, N., \& Singh, A. (2009). A study on farmers' knowledge, perception and intensity of pesticide use in vegetable cultivation in Western Uttar Pradesh.

[7] Manandhar, D. N., \& Manandhar, S. D. (2006). Pesticide Issues in Print Media. Thamel, Galkopakha. 155-160 .

[8] Mills, P. K., \& Kwong, S. (2001). Cancer incidence in the United Farmworkers of America (UFW), 19871997. American Journal of Industrial Medicine, 40(5), 596-603.

[9] Palikhe B. R. (2002). "Challenges and options of pesticide use: in the context of Nepal. 
" Landschaftsökologie und Umweltforschung" 38: 130-

141

[10] Pimentel, D., McLaughlin, L., Zepp, A., Lakitan, B., Kraus, T., Kleinman, P., \& Selig, G. (1993). Environmental and economic effects of reducing pesticide use in agriculture. In Agriculture and the Environment, 273-288.

[11] Ransom P. (2002). "Women, pesticides and sustainable agriculture." Women's Caucus for the Earth Summit, CSD NGO Women's Caucus.

[12] Recena, M. C. P., Caldas, E. D., Pires, D. X., \& Pontes, E. R. J. (2006). Pesticides exposure in Culturama, Brazil knowledge, attitudes, and practices. Environmental Research, 102(2), 230-236.

[13] Sharma, D. R., Thapa, R. B., Manandhar, H. K., Shrestha, S. M., \& Pradhan, S. B. (2012). Use of pesticides in Nepal and impacts on human health and environment. Journal of Agriculture and Environment, 13, 67-74.

[14] Shrestha P., Koirala P., and Tamrakar A. S. (2010). "Knowledge, Practice and Use of Pesticides among Commercial vegetable Growers of Dhading District, Nepal." Journal of Agriculture and Environment 11 : 95-100.

[15] Tingle, C. C. D., Rother, J. A., Dewhurst, C. F., Lauer, S., \& King, W. J. (2000). Health and environmental effects of fipronil. Briefing paper for Pesticides Action Network, UK.

[16] Weston, D. P., You, J., \& Lydy, M. J. (2004). Distribution and toxicity of sediment-associated pesticides in agriculture-dominated water bodies of California's Central Valley. Environmental Science \& Technology, 38(10), 2752-2759.

[17] Wilson C. and Tisdell C. (2001). "Why farmers continue to use pesticides despite environmental, health and sustainability costs." Ecological Economics 39.3: 449-462. 